\title{
Analysis of cytolytic capability of natural killer cells and cytotoxic T cells in the peripheral blood of cancer patients
}

\author{
Ismail Atia Hassan \\ Zoology Department, Faculty of Science, Al-Azhar University, Assiut Branch, Assiut, Egypt
}

\section{III}

Background: Lung cancer is a one of the most cancer types speeded worldwide. In Egypt, lung cancer counted as the third cause of deaths among males after liver and bladder cancers. It is characterized with two type's SCLC represents about (10\% to $15 \%$ ) and NSCLCs which represents ( $80 \%$ to $85 \%$ ). In NSCLCs, treatment only treat $15 \%-30 \%$ of patients due to the presence of tumor mediators which enhance tumor surveillance by secreting PGE2, TGF- $\beta$. CD8+ and NK are the main cells to defeat and kill cancer cells. Aim: The aim of this study is to address the functionality of T-cells and NK cells from lung cancer patient's pre and post stimulation in-vitro. Lung cancer is a one of the most cancer types speeded worldwide. In Egypt, lung cancer counted as the third cause of deaths among males after liver and bladder cancers. Lung cancer characterized with two type's SCLC represents about (10\% to 15\%) and NSCLCs which represents (80\% to $85 \%$ ). In NSCLCs, treatment only treat $15 \%-30 \%$ of patients due to the presence of tumor mediators which enhance tumor surveillance by secreting PGE2, TGF- $\beta$. CD8+ and NK are the main cells to defeat and kill cancer cells. The aim of this study is to address the functionality of T-cells and NK cells on lung cancer patient's pre and post stimulation in-vitro. Materials and methods. 